\title{
Accuracy of Cardiac Innervation Scintigraphy for Mild Cognitive Impairment With Lewy Bodies
}

Gemma Roberts, PhD, Rory Durcan, MRCPI, MSc, Paul C. Donaghy, PhD, Sarah Lawley, MBBS, Joanna Ciafone, PhD, Calum A. Hamilton, MSc, Sean J. Colloby, PhD, Michael J. Firbank, PhD, Louise Allan, PhD, Nicola Barnett, MSc, Sally Barker, BSc, Kim Howe, BSc, Tamir Ali, FRCR, George S. Petrides, FRCR, Jim Lloyd, PhD, John-Paul Taylor, PhD, John O'Brien, DM, and Alan J. Thomas, PhD

Neurology ${ }^{\circledR}$ 2021;96:e2801-e2811. doi:10.1212/WNL.0000000000012060

\section{Abstract \\ Objective}

To provide evidence that cardiac I-123-metaiodobenzylguanidine sympathetic innervation imaging (MIBG) scintigraphy differentiates probable mild cognitive impairment with Lewy bodies (MCI-LB) from mild cognitive impairment due to Alzheimer disease (MCI-AD), we scanned patients with MCI and obtained consensus clinical diagnoses of their MCI subtype. We also performed baseline FP-CIT scans to compare the accuracy of MIBG and FP-CIT.

\section{Methods}

We conducted a prospective cohort study into the accuracy of cardiac MIBG scintigraphy in the diagnosis of MCI-LB. Follow-up clinical assessment was used to diagnose MCI-AD (no core features of MCI-LB and normal FP-CIT), probable MCI-LB ( 2 or more core features, or 1 core feature with abnormal FP-CIT), or possible MCI-LB ( 1 core feature or abnormal FP-CIT). For the comparison between MIBG and FP-CIT, only core clinical features were used for diagnosis.

\section{Results}

We recruited 95 people with mild cognitive impairment. Cardiac MIBG was abnormal in 22/37 probable and 2/15 possible MCI-LB cases and normal in 38/43 MCI-AD cases. The sensitivity in probable MCI-LB was 59\% (95\% confidence interval [CI], 42\%-75\%), specificity $88 \%$ (75\%-96\%), and accuracy 75\% (64\%-84\%). The positive likelihood ratio was 5.1 and negative likelihood ratio 0.46 . With symptom-only diagnoses, the accuracies were $79 \%$ for MIBG (95\% CI, $68 \%-87 \%$ ) and $76 \%$ for FP-CIT (95\% CI, 65\%-85\%).

\section{Conclusions}

Cardiac MIBG appears useful in early disease, with an abnormal scan highly suggestive of MCILB. Validation in a multicenter setting is justified.

\section{Classification of Evidence}

This study provides Class I evidence that cardiac MIBG distinguishes MCI-LB from MCI-AD.

\author{
Correspondence \\ Dr. Roberts \\ Gemma.roberts@ \\ newcastle.ac.uk
}

\section{MORE ONLINE}

III Class of Evidence

Criteria for rating

therapeutic and diagnostic

studies

NPub.org/coe 


\section{Glossary}

ACE-R = Addenbrooke's Cognitive Examination-Revised; AD = Alzheimer disease; CDR = Clinical Dementia Rating; $\mathbf{C I}=$ confidence interval; DLB = dementia with Lewy bodies; GDS = Geriatric Depression Scale; HMR = heart-to-mediastinum count ratio; IADL = Instrumental Activities of Daily Living; LB = Lewy bodies; LBD = Lewy body disease; $\mathbf{M C I}=$ mild cognitive impairment; MCI-AD = mild cognitive impairment with Alzheimer disease; MCI-LB = mild cognitive impairment with Lewy bodies; MI = myocardial infarction; MIBG = I-123-metaiodobenzylguanidine; MMSE = Mini-Mental State Examination; NIA-AA = National Institute on Aging-Alzheimer's Association; NPI = Neuropsychiatric Inventory; SNRI = serotonin-norepinephrine reuptake inhibitor; SSRI = selective serotonin reuptake inhibitor; UPDRS = Unified Parkinson's Disease Rating Scale.

Accurate biomarkers for diagnosing early-stage neurodegenerative diseases, such as Alzheimer disease (AD) and Lewy body disease (LBD), are urgently required so that future disease-modifying treatments can be given early on and prevent deterioration to dementia. Recent unsuccessful reports of new $\mathrm{AD}$ treatments may be related to them being given late in the disease course and to people with pathology other than pure $\mathrm{AD} .{ }^{1} \mathrm{LBD}$ is recognized as a common copathology even in well-characterized $\mathrm{AD}$ cohorts $^{2}$ but can be challenging to detect. Cardiac I-123-metaiodobenzylguanidine sympathetic innervation imaging (MIBG) is included as an indicative biomarker in the fourth dementia with Lewy bodies (DLB) consensus criteria, alongside dopaminergic imaging and polysomnography. ${ }^{3}$ Cardiac MIBG is well-established as a diagnostic marker in DLB, with good sensitivity (70\%) and specificity (90\%), but is much less studied at the mild cognitive impairment (MCI) stage (probable MCI with Lewy bodies $[\mathrm{MCI}-\mathrm{LB}])$. The recent consensus research criteria for MCI-LB ${ }^{4}$ emphasize the need for prospective studies to assess the diagnostic accuracy at MCI stages.

Our previous study assessing dopaminergic imaging in MCI$\mathrm{LB}^{5}$ confirmed a high specificity of $89 \%$ (95\% confidence interval [CI], 71\%-98\%), similar to DLB. Sensitivity for probable MCI-LB was lower than in DLB at 61\% (95\% CI, 43\%-77\%). We hypothesized that cardiac sympathetic innervation imaging with MIBG would differentiate between probable MCI-LB and $\mathrm{MCI}$ with probable $\mathrm{AD}$ (MCI-AD) cases.

\section{Methods}

\section{Study Design}

We conducted a single-center prospective cohort study into the accuracy of planar cardiac MIBG scintigraphy in the diagnosis of MCI-LB. We report the results here using the Enhancing the Quality and Transparency of Health Research (EQUATOR) network Standards for Reporting of Diagnostic Accuracy (STARD) guidelines for diagnostic accuracy studies. ${ }^{6}$ Our index test is the dichotomized cardiac MIBG uptake result (heart to mediastinum ratio; see Image Acquisition and Processing). Our reference standard is consensus diagnosis of MCI-LB or $\mathrm{MCI}-\mathrm{AD}$, which was based on the most recent core features and FP-CIT imaging result (see Clinical Diagnosis).
Our primary research question was as follows: What is the accuracy of cardiac MIBG scintigraphy for the differential diagnosis of probable MCI-LB vs MCI-AD? This study provides Class I evidence relating to this research question.

We also explored the diagnostic performance in the less certain diagnostic category of possible MCI-LB, and with possible and probable $\mathrm{MCI}$ combined as a single diagnostic group.

We considered the relationship and concordance between cardiac MIBG and FP-CIT results in patients with suspected MCI-LB as a secondary analysis, recognizing the study was not designed to provide statistical power for a full comparison of the diagnostic accuracy of the 2 biomarkers.

\section{Patient Recruitment}

Patients aged 60 or older with a clinical diagnosis of MCI were recruited from local memory services in the north-east of England between April 2016 and September 2019. The medical records of all patients meeting the above criteria were reviewed to assess eligibility. In addition to the diagnosis of MCI, we searched their health service records for evidence of any clinical features indicating they may have had Lewy body (LB) disease, for example, autonomic dysfunction, hyposmia, sleep disturbance, or 1 or more core clinical symptoms of DLB., Following the medical research assessment, the consensus clinical diagnosis was based on the presence or absence of the 4 core features (visual hallucinations, fluctuations, parkinsonism, and REM sleep behavior disorder; see Clinical Diagnosis). Thus, nonspecific symptoms such as falls, hyposmia, anxiety, depression, and constipation were not used to define the clinical groups. Following informed consent, participants underwent a research interview, clinical assessment, and neurologic examination by a medical doctor (R.D., S.L.). Clinical assessment included the following tests and rating scales: the Movement Disorders Society-Unified Parkinson's Disease Rating Scale motor examination (UPDRS-III), Epworth Sleepiness Scale, Geriatric Depression Scale (GDS), Instrumental Activities of Daily Living (IADL) scale, Neuropsychiatric Inventory (NPI), and Clinical Dementia Rating (CDR). ${ }^{7}$ A detailed neuropsychological evaluation included Addenbrooke's Cognitive Examination-Revised (ACE-R) and Mini-Mental State Examination (MMSE), as reported previously. ${ }^{7}$ All patients were offered dopaminergic imaging with ${ }^{123}$ I-FP-CIT SPECT at 
Figure 1 Participant Flowchart Showing Resulting Number of Normal and Abnormal Cardiac I-123-metaiodobenzylguanidine (MIBG) Scans in the Mild Cognitive Impairment With Alzheimer Disease (MCI-AD) and Mild Cognitive Impairment With Lewy Bodies (MCI-LB) Groups

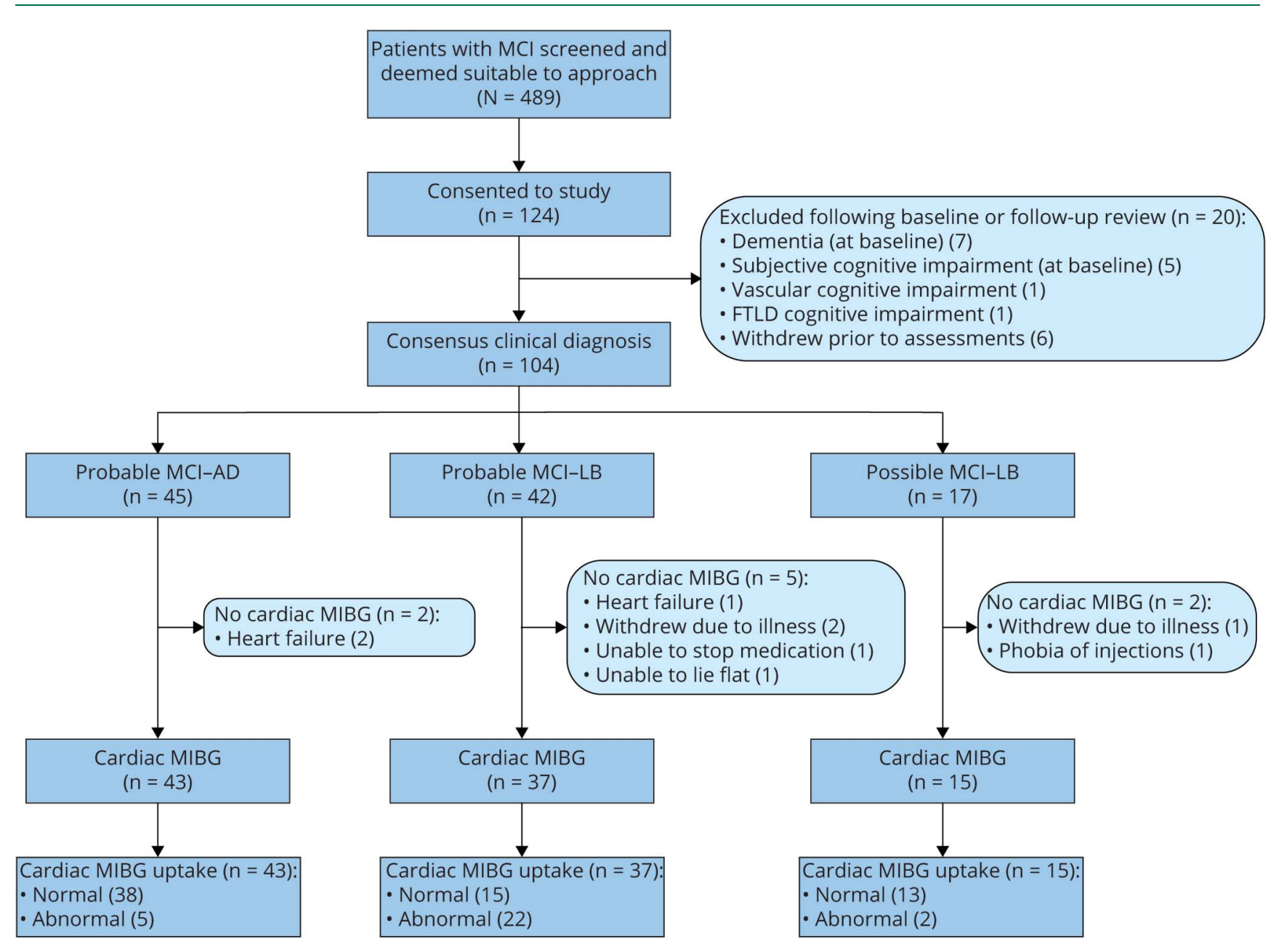

FTLD = frontotemporal lobar degeneration; $\mathrm{MCl}=$ mild cognitive impairment.

baseline, if not done clinically within the past 12 months, in which case these images were used with permission. Images were assessed as normal or abnormal by an experienced consensus panel. This was done blind to all clinical and diagnostic information, as reported earlier. ${ }^{5}$

\section{Exclusion Criteria}

Patients with dementia rather than MCI, vascular or frontotemporal etiologies, history of stroke, or parkinsonism predating cognitive impairment by more than 1 year were excluded. Published guidance focusses on the use of cardiac MIBG for cardiology applications and there are currently no European or US guidelines for the use of cardiac MIBG imaging in LBD. However, cardiac MIBG is used in many other conditions-heart failure, myocardial infarction (MI), ischemic heart disease, diabetes mellitus, atrial fibrillation-these conditions can therefore be assumed to have the potential to reduce uptake if present in our patients so a detailed medical history was recorded. We excluded patients with heart failure
(New York Heart Association Class II or worse), as this can cause global sympathetic cardiac denervation. We excluded patients with MI within a year prior to recruitment, assuming that reinnervation of the infarcted region occurs within that timeframe. We excluded participants taking tramadol, labetalol, or tricyclic antidepressants (other than $<10 \mathrm{mg}$ amitriptyline) if they were not able to withdraw 48 hours prior to the cardiac MIBG scan, broadly in line with the recommendations of Jacobson and Travin ${ }^{8}$ in their 2015 review of the literature. Selective serotonin reuptake inhibitors (SSRIs), serotoninnorepinephrine reuptake inhibitors (SNRIs), and calcium channel blockers were permitted but recorded for later review.

\section{Standard Protocol Approvals, Registrations, and Patient Consents}

All participants gave their written informed consent to take part in the study. The study received ethical approval from an ethical standards committee on human experimentation: the National Research Ethics Service Committee North East Newcastle \& 
Table 1 Demographic and Clinical Data for the Mild Cognitive Impairment With Alzheimer Disease (MCI-AD), Mild Cognitive Impairment With Lewy Bodies (MCI-LB), and Control Groups

\begin{tabular}{|c|c|c|c|c|c|}
\hline & MCI-AD & Probable MCI-LB & Possible MCl-LB & Controls & $p$ Value \\
\hline $\mathbf{N}$ & 43 & 37 & 15 & 30 ( 2 excluded) & NA \\
\hline Female & $25(58)$ & $2(5)$ & $6(40)$ & $8(27)$ & $<0.001$ \\
\hline Age at consent & $75.3(7.5)$ & $74.8(6.1)$ & $73.7(7.4)$ & $73.8(7.2)$ & 0.78 \\
\hline Follow-up, y & $1.1(1.0)$ & $1.6(0.85)$ & $1.1(1.3)$ & $1.3(0.61)$ & 0.03 \\
\hline BMI & $26.4(4.8)$ & $27.0(4.7)$ & $29.1(5.5)$ & $28.2(5.2)$ & 0.60 \\
\hline UPDRS total & $14.4(13.3)$ & $23.5(14.5)$ & $15.1(9.2)$ & $5.5(4.3)$ & 0.004 \\
\hline MMSE & $27.0(2.1)$ & $26.6(2.4)$ & $25.7(2.9)$ & $28.3(1.1)$ & 0.51 \\
\hline ACE total & $82.9(8.4)$ & $83.5(9.3)$ & $77.0(11.9)$ & $92.1(4.1)$ & 0.78 \\
\hline Epworth Sleepiness Scale & $5.5(4.2)$ & $8.8(4.2)$ & $5.3(4.0)$ & $4.5(3.1)$ & 0.01 \\
\hline Geriatric Depression Scale & $3.4(2.7)$ & $4.6(3.9)$ & $4.3(3.8)$ & $1.1(1.8)$ & 0.20 \\
\hline IADL & $7.2(1.3)$ & $6.3(1.4)$ & $6.3(1.7)$ & NA & 0.002 \\
\hline CDR & $0.5(0.0)$ & $0.5(0.1)$ & $0.5(0.1)$ & $0.0(0.0)$ & 0.14 \\
\hline NPI & $8.9(10.3)$ & $16.1(12.4)$ & $12.8(14.7)$ & NA & 0.01 \\
\hline Memantine & $1(2)$ & $1(3)$ & $2(13)$ & NA & 0.94 \\
\hline Cholinesterase inhibitor & $8(19)$ & $19(51)$ & $2(13)$ & NA & 0.003 \\
\hline Antiparkinsonian drug & 0 & $4(11)$ & 0 & NA & 0.03 \\
\hline Fluctuations (baseline) & 0 & $20(54)$ & $2(13)$ & NA & $<0.001$ \\
\hline Visual hallucinations (baseline) & 0 & $6(16)$ & $2(13)$ & NA & 0.03 \\
\hline Parkinsonism (baseline) & 0 & $13(35)$ & $1(7)$ & NA & $<0.001$ \\
\hline REM sleep behavior disorder (baseline) & 0 & $29(78)$ & $3(20)$ & NA & $<0.001$ \\
\hline
\end{tabular}

Abbreviations: $\mathrm{ACE}=$ Addenbrooke's Cognitive Examination; $\mathrm{BMI}=$ body mass index; $\mathrm{CDR}=$ Clinical Dementia Rating; IADL = instrumental activities of daily living; MMSE = Mini-Mental State Examination; NPI = Neuropsychiatric Inventory; UPDRS = Unified Parkinson's Disease Rating Scale.

Values are mean (SD) or $\mathrm{n}(\%) . p$ Values are between the probable MCI-AD and MCI-LB groups.

North Tyneside 2 (research ethics committee identification number 15/NE/0420). All data are available on the Dementias Platform UK (DPUK) upon reasonable request.

\section{Clinical Diagnosis}

Three experienced board-certified old age psychiatrists (A.J.T., P.C.D., J.-P.T.) each independently reviewed the research assessment clinical documentation and confirmed diagnoses of MCI according to National Institute on Aging-Alzheimer's Association (NIA-AA) criteria. ${ }^{9}$ This method is validated against autopsy and recognized as the clinical gold standard for living patients. ${ }^{10,11} \mathrm{MCI}$ was confirmed if there was evidence of minimal functional impairment and a CDR of 0 or 0.5 , and a history of subjective and objective cognitive decline from assessment and health service records.

To determine whether the MCI was likely due to LBD, the presence of each of the 4 core LB features was determined by the same panel of old age psychiatrists (A.J.T., P.C.D., J.-P.T.) following the fourth consensus criteria for DLB. ${ }^{3}$ For most participants, annual review data were available to the consensus panel, as well as the baseline research assessments. Where possible, a spouse or other family member was interviewed to provide additional information. To strengthen the diagnostic groupings, FP-CIT results were later incorporated into diagnoses alongside the presence of core LB features, in accordance with consensus criteria. The consensus decision on the presence or absence of the core clinical features was done without reference to the FP-CIT images. The latest FP-CIT result was then combined with the number of core features at follow-up to obtain the consensus diagnoses. At the time of assessment, the panel was blind to both FP-CIT and MIBG results.

MCI-LB was diagnosed in patients with either 2 or more core LB features (including those with normal FP-CIT) or 1 or more core features and abnormal dopaminergic imaging. Patients were grouped as possible MCI-LB if they presented with only 1 core feature with normal FP-CIT or abnormal FPCIT with no core features. Participants were grouped as MCI$\mathrm{AD}$ when they had none of the 4 core $\mathrm{LB}$ features, a normal 
Figure 2 Heart-to-Mediastinum Count Ratio (HMR) Results for Probable Mild Cognitive Impairment With Alzheimer Disease (MCI-AD), Probable Mild Cognitive Impairment With Lewy Bodies (MCI-LB), and Controls, With the HMR Cutoff of 1.85 Shown as a Solid Line
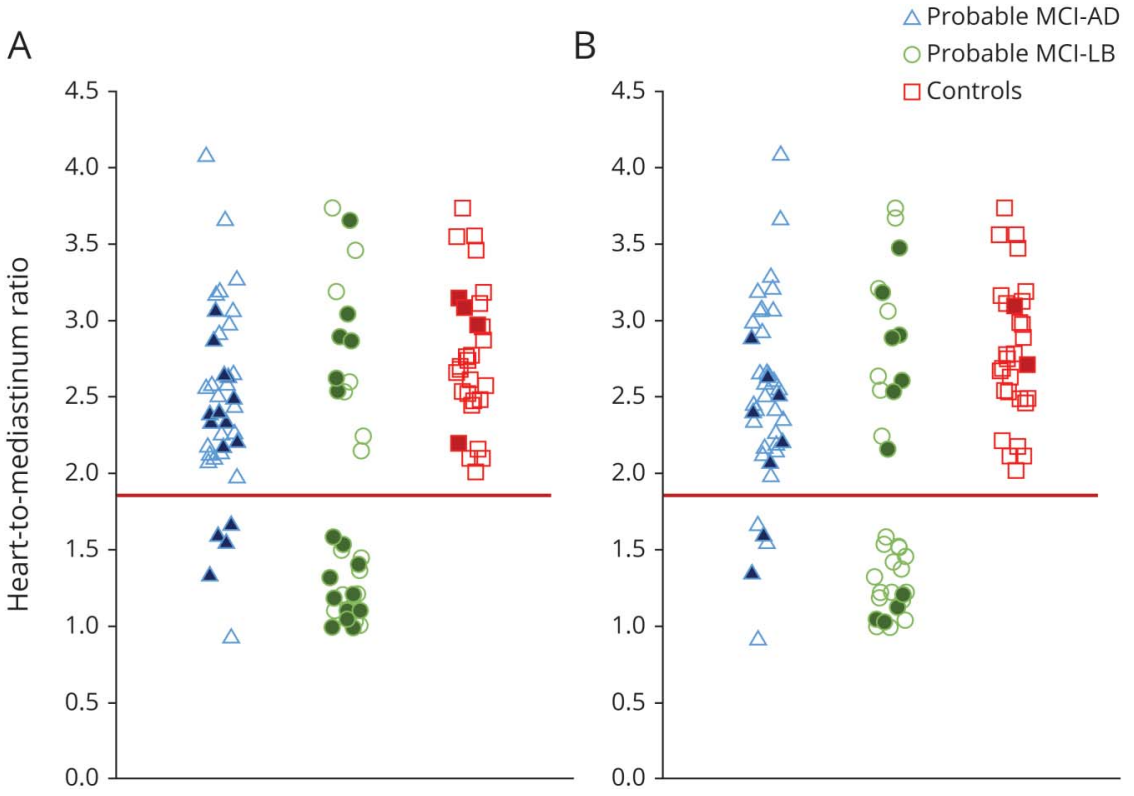

(A) Filled markers = type 2 diabetes or heart conditions. (B) Filled markers = potentially interacting medications.

FP-CIT scan, and decline characteristic of $\mathrm{AD}$, that is, fulfilling the NIA-AA criterion of "etiology of MCI consistent with $\mathrm{AD}$ pathophysiologic process." 9 This makes $\mathrm{AD}$ the most likely cause, although other non-LB causes cannot be excluded. $A D$-specific biomarkers were not used as we aimed to exclude $\mathrm{LB}$ disease rather than confirm $\mathrm{AD}$ pathology.

\section{Controls Without Cognitive Impairment}

Thirty-two individuals aged over 60 years with normal cognition were recruited from participants' spouses and people who responded to advertising on a national website. They underwent a detailed neurologic examination by a medical doctor $(\mathrm{RD}, \mathrm{SL})$, including rating for parkinsonism with the UPDRS, the same thorough neurocognitive examination as for MCI participants, confirming normal cognition, and an MRI brain scan within normal limits.

\section{Image Acquisition and Processing}

\section{Cardiac I-123-MIBG}

Imaging took place within 1 month of baseline clinical assessment. All individuals were administered $111 \mathrm{MBq}$ ( $\pm 10 \%)$ I-123MIBG via slow IV injection. Ten-minute anterior planar images were acquired between 3.5 and 4.5 hours after injection. All images were acquired on a Siemens gamma camera (Siemens Healthcare) with medium energy low penetration collimators.

Cardiac MIBG uptake was quantified using the heart-tomediastinum count ratio (HMR) as a diagnostic indicator, as described in our previous publication, ${ }^{12}$ which includes example images showing cardiac and mediastinum region placement.
Normal HMR values depend on both technical factors and the patient population, as detailed in our previous work. ${ }^{13}$ A local HMR cutoff value was therefore derived from the control images, and set as 2 SDs below the mean HMR value.

\section{${ }^{123}$ I-FP-CIT SPECT \\ ${ }^{123}$ I-FP-CIT scans were also done within 1 month of baseline assessment, unless a scan had been acquired for clinical rea- sons within the previous 6 months, in which case it was not repeated, in accordance with ethical approval. This was the case for 4 patients, whose images were acquired using a similar protocol to the study scan protocol.}

Three to 6 hours following a bolus IV injection of $185 \mathrm{MBq}$ of ${ }^{123}$ I-FP-CIT (Ioflupane [DaTSCAN], GE Healthcare), patients were scanned using a double-headed Siemens gamma camera fitted with a low energy high resolution parallel hole collimator.

Images were reconstructed using iterative reconstruction with resolution recovery, uniform attenuation correction, and Monte Carlo scatter correction. For all images, transverse data were manually reoriented to adjust for head tilt during reconstruction.

Visual assessment of all scans was undertaken by 5 experienced raters blind to clinical diagnosis and information, using an established FP-CIT rating scale, ${ }^{14}$ shown to be accurate in the differential diagnosis of $\mathrm{DLB}$ and $\mathrm{AD} .{ }^{15}$ Semiquantification results from BRASS (Hermes Medical Solutions) were available to the raters. After panel discussions, a consensus normal or abnormal was obtained for each participant. 
Table 2 Patients Taking Medications Suspected of Potentially Interacting With Cardiac I-123-metaiodobenzylguanidine (MIBG) Uptake and Percentages With History of Medical Conditions Known to Affect MIBG Uptake

\begin{tabular}{|c|c|c|c|c|}
\hline & MCI-AD & Probable MCI-LB & Possible MCI-LB & Controls \\
\hline $\mathbf{N}$ & 43 & 37 & 15 & 30 \\
\hline \multicolumn{5}{|l|}{ Potentially interacting medications } \\
\hline Amlodipine & $7(16)$ & $8(22)$ & $3(20)$ & $4(13)$ \\
\hline Nifedipine & $1(2)$ & $1(3)$ & 0 & 0 \\
\hline Lercanidipine & $1(2)$ & 0 & $1(7)$ & $1(3)$ \\
\hline Diltiazem & $1(2)$ & $1(3)$ & 0 & 0 \\
\hline Salbutamol & $1(2)$ & $3(8)$ & $1(7)$ & $3(10)$ \\
\hline Tramadol & $1(2)$ & $2(5)$ & $1(7)$ & $1(3)$ \\
\hline Amitriptyline $\leq 25 \mathrm{mg}$ & $4(9)$ & 0 & $1(7)$ & $2(7)$ \\
\hline Mirtazapine & $3(7)$ & $4(11)$ & 0 & $1(3)$ \\
\hline Duloxetine & $1(2)$ & $2(5)$ & $1(7)$ & 0 \\
\hline \multicolumn{5}{|l|}{ Relevant medical history } \\
\hline Type 2 diabetes mellitus & $7(16)$ & $6(16)$ & $4(27)$ & $3(10)$ \\
\hline Ischemic heart disease/angina & $5(12)$ & $8(22)$ & $1(7)$ & 0 \\
\hline Myocardial infarction & $3(7)$ & $8(22)$ & $1(7)$ & $1(3)$ \\
\hline Atrial fibrillation & $4(9)$ & $2(5)$ & $2(13)$ & $1(3)$ \\
\hline Heart failure & 0 & 0 & 0 & 0 \\
\hline
\end{tabular}

Abbreviations: MCI-AD = mild cognitive impairment with Alzheimer disease; MCI-LB = mild cognitive impairment with Lewy bodies.

Values are $\mathrm{n}(\%)$.

Participants whose status remained as MCI were invited to undergo a repeat FP-CIT scan 1-2 years after baseline and 51 participants with MCI completed this. The most recent FP-CIT scan result was used when incorporating the results into the diagnostic criteria for MCI-LB, but the baseline FP-CIT result was used in the comparison between FP-CIT and MIBG diagnostic accuracy.

\section{Statistical Analysis}

The target sample size for the probable MCI-LB and MCI-AD groups was 41 patients per group, to estimate diagnostic accuracy of MIBG with $95 \%$ confidence to within \pm 10 percentage points, determined using data from our previous MCI-LB study in FPCIT. Our principal aim was not to compare the diagnostic accuracy of MIBG and FP-CIT, and thus the present study was not powered to address this question. However, knowing the interest such a comparison holds for the field, we report exploratory findings comparing these 2 biomarkers to help inform sample sizes that would be needed for a head-to-head comparison.

Statistical Package for Social Sciences software (SPSS version 25) was used to produce summary statistics. Continuous variables were analyzed for difference between the MCI-AD and probable MCI-LB groups using the Student $t$ test for independent samples, where histograms showed an approximate normal distribution (age, body mass index, MMSE, ACE, extrapyramidal symptoms).
The Levene test was used to determine whether equal variance could be used. The Mann-Whitney $U$ test was used if the histograms of the MCI-AD or MCI-LB groups appeared skewed (follow-up, UPDRS, GDS, IADL, CDR, NPI). The $\chi^{2}$ test was used for determining whether there was a difference in the proportions of binary variables.

The sensitivity, specificity, and overall accuracy of cardiac MIBG as a biomarker for probable MCI-LB (values) was calculated from a $2 \times 2$ frequency table, applying exact binomial 95\% CIs for proportions obtained from statpages. info/confint.html. Likelihood ratios were calculated to estimate the added value of cardiac sympathetic innervation imaging in the diagnosis of probable MCI-LB.

Table 3 I-123-metaiodobenzylguanidine (MIBG) and FPCIT Concordance for Patients With 1 or More Lewy Body Core Symptoms

\begin{tabular}{llll}
\hline & MIBG normal & MIBG abnormal & Totals \\
\hline FP-CIT normal & 19 & 6 & 25 \\
\hline FP-CIT abnormal & 5 & 15 & 20 \\
\hline Totals & 24 & 21 & 45 \\
\hline
\end{tabular}


Figure 3 Example of Probable Mild Cognitive Impairment With Lewy Bodies (MCl-LB) With Mismatched FPCIT and Cardiac I-123-metaiodobenzylguanidine (MIBG) Results

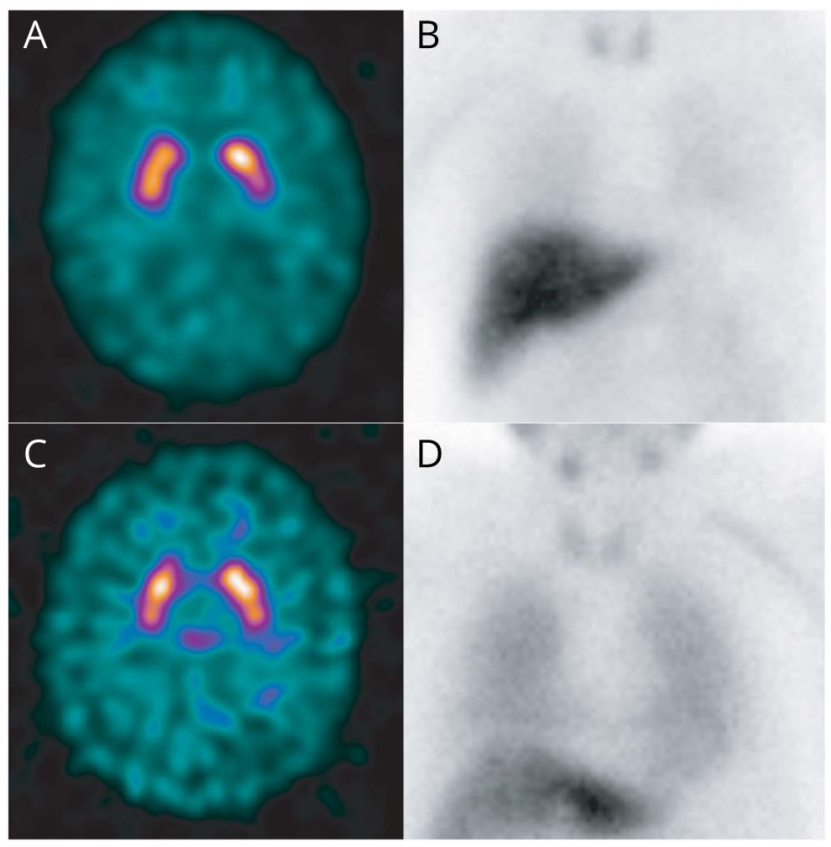

The top row is from a patient with (A) normal baseline FP-CIT and (B) abnormal cardiac MIBG with no visible uptake in the myocardium and heart-to-mediastinum count ratio (HMR) of 1.44. The bottom row is a patient with (C) baseline FPCIT rated as abnormal and (C) normal cardiac MIBG with HMR of 2.23.

In our secondary analysis, focusing on the relationship between cardiac MIBG and FP-CIT results, we used consensus clinical diagnosis based on the presence of the 4 core clinical features only without indicative biomarkers. This enabled an initial comparison between FP-CIT and MIBG, recognizing that the study was not designed to power this comparison. In this secondary analysis, possible MCI-LB therefore means the presence of 1 core feature (fluctuations, visual hallucinations, REM sleep behavior disorder, or parkinsonism) at the most recent clinical assessment by the study team, and probable MCI-LB means the presence of 2 or more of the core features. For this comparison, we excluded the 1 patient with MCI-AD who did not have FPCIT, so that the same groups were used for accuracy calculation for both MIBG and FP-CIT. We assessed the concordance between MIBG and FP-CIT results for each diagnostic group. Cohen kappa was used to assess the agreement between MIBG and FP-CIT for patients with 1 or more core LB feature. We assessed the benefit of combining MIBG and FP-CIT results by calculating sensitivities and specificities when either scan can be abnormal for a positive test result and when both scans must be abnormal for a positive result.

As a further analysis, we calculated the proportion of patients with probable MCI-LB with positive MIBG scans and the proportion with positive FP-CIT scans for each of the core features. Fisher exact test was used to test for any difference in proportions. This was to explore whether any symptom profile would favor choosing one scan over another.

\section{Data Availability}

Data are available from the lead author by email on reasonable request.

\section{Results}

After initial screening, 124 eligible patients consented to take part, although 20 patients were later excluded or withdrew (see flow chart in figure 1). Our final groups consisted of 37 probable MCI$\mathrm{LB}, 15$ possible MCI-LB, and $43 \mathrm{MCI}-\mathrm{AD}$ (figure 1). One patient did not attend for FP-CIT so the result could not be used as part of the consensus diagnosis for this patient. This patient had none of the 4 core clinical symptoms of MCI-LB. No adverse effects from the cardiac MIBG scan or FP-CIT were reported. The demographic and clinical characteristics of the MCI groups and controls are given in table 1 , with $p$ values for difference between MCI-AD and probable MCI-LB groups. As expected, there was a higher proportion of men in the MCI-LB group.

Two control volunteers with scans demonstrating abnormal cardiac MIBG uptake were excluded from analysis to determine local normal HMR cutoffs; our control group therefore consisted of 30 volunteers. These individuals had no significant medical history and were not taking any medications suspected of interfering with cardiac MIBG uptake. The control group HMR values resulted in an HMR cutoff of 1.85 (calculated from the unrounded mean and SD values). The 2 excluded volunteers had HMR results around 3 SDs below the mean $(z$ scores -2.8 and -3.7 ).

Our primary outcome was the diagnostic accuracy of cardiac MIBG scintigraphy for the differential diagnosis of probable MCI-LB from MCI-AD. The sensitivity for detecting probable MCI-LB was 59\% (95\% CI, 42\%-75\%), specificity $88 \%$ (75\%-96\%), and accuracy $75 \%(64 \%-84 \%)$. The positive likelihood ratio was 5.1 and negative likelihood ratio 0.46 . The percentages of abnormal scans in the probable MCI-LB and MCI-AD groups were $59 \%$ and $12 \%$, respectively; this difference in proportions of abnormal MIBG scans was statistically significant $(p<0.001)$.

Combining the probable and possible MCI-LB into a single LB group gave an overall sensitivity of $46 \%$ (95\% CI, $32 \%-61 \%$ ).

The mean (SD) HMR ratios are as follows: controls: 2.76 (0.45); MCI-AD: 2.48 (0.59); probable MCI-LB: 1.89 (0.88); possible MCI-LB: $2.64(0.67)$. The difference in the mean HMR between the probable MCI-LB and MCI-AD group was $0.60(p=0.001,95 \%$ CI, 0.25-0.95).

Dot plots of these results for probable MCI-LB, MCI-AD, and controls, with the HMR cutoff of 1.85 illustrated, are shown in 
Table 4 Patients With Mild Cognitive Impairment With Lewy Bodies With Positive I-123metaiodobenzylguanidine (MIBG) or Positive FPCIT Scans for Each of the 4 Core Lewy Body Features

\begin{tabular}{lccll}
\hline & N & MIBG+ & FP-CIT+ & $\begin{array}{l}\boldsymbol{p} \text { Value } \\
\text { (Fisher } \\
\text { exact test) }\end{array}$ \\
\hline Fluctuations (baseline) & 18 & $12(67)$ & $10(56)$ & 0.73 \\
\hline Hallucinations (baseline) & 5 & $4(80)$ & $5(100)$ & 1.00 \\
\hline Parkinsonism (baseline) & 12 & $11(92)$ & $11(92)$ & 1.00 \\
\hline RBD (baseline) & 25 & $15(60)$ & $14(56)$ & 1.00 \\
\hline
\end{tabular}

Abbreviation: RBD = REM sleep behavior disorder.

Values are $\mathrm{n}(\%)$.

figure 2. The patients with heart conditions (including previous MI) or type 2 diabetes mellitus and those taking potentially interacting medications (SSRIs, SNRIs, or low dose $[<25 \mathrm{mg}]$ amitriptyline) are shown. Table 2 details the medical conditions and potentially interacting medication for each group. The numbers are too low for meaningful formal statistical analysis, but do not show any clear influence of interacting medications or comorbidities on cardiac uptake for any of the groups.

One patient was excluded from the comparison between MIBG and baseline FP-CIT results because they did not attend for FPCIT scanning. A further patient's FP-CIT scan was excluded because the scan was highly asymmetric and MRI showed evidence of infarct. The recategorized diagnostic groups based on the latest core symptoms only consisted of $48 \mathrm{MCI}-\mathrm{AD}$ (no core symptoms), 17 possible MCI-LB (1 core symptom), and 28 probable MCI-LB (2+ core symptoms).

Of the probable MCI-LB group (2+ core symptoms), 17/28 patients had abnormal MIBG scans and the same proportion had abnormal FP-CIT scans, although not all in the same patients. This results in a sensitivity of $61 \%$ (95\% CI, $41 \%-79 \%)$ for both MIBG and FP-CIT. Of the MCI-AD group (no core symptoms), 43/48 patients had normal MIBG scans and 41/48 had normal FP-CIT scans, resulting in similar specificities of $90 \%$ for MIBG (95\% CI, 77\%-97\%) and $85 \%$ for FP-CIT (95\% CI, 72\%-94\%). The overall accuracy is $79 \%$ for MIBG (95\% CI, $68 \%-87 \%$ ) and $76 \%$ for FPCIT (95\% CI, 65\%-85\%).

By combining the 2 tests and taking a positive result as 1 or both tests abnormal, the sensitivity increases from $61 \%$ to $71 \%$. However, the specificity drops from $90 \%$ (MIBG only) to $75 \%$. The specificity is $100 \%$ if 2 abnormal scans are required for a positive result. This reduces the sensitivity from $59 \%$ to $50 \%$.

Although the current study is not powered to determine whether the difference in accuracy between MIBG and FP-CIT is significant, we examined the cases with LB symptoms for which the MIBG and FP-CIT results are mismatched. This was the case for $5 / 17$ patients with 1 core symptom (29\%) and 6/28 patients with 2 or more core symptoms (21\%) (table 3 ). Example images for patients with probable MCI-LB with mismatched FP-CIT and cardiac MIBG results are given in figure 3. Cohen kappa was 0.57 , indicating fair agreement between FP-CIT and MIBG for patients with 1 or more core features of LBD. Several of the patients with mismatches had difficult FP-CIT scans to rate, with the panel in full agreement prior to discussion in only $4 / 11$ cases. Only 1 of the MIBG results could be considered borderline, with $\mathrm{HMR}=1.76$. This patient had fluctuations only at latest assessment, and a clear normal baseline and follow up FP-CIT scan. There was no obvious pattern between the presence of individual core clinical symptoms at baseline or latest assessment and whether the MIBG or FP-CIT was abnormal. None of the patients were on any medications affecting striatal DaT binding. ${ }^{16}$ One patient with a positive baseline FP-CIT scan and normal MIBG at baseline had a normal FP-CIT scan at followup, suggesting a false-positive or equivocal baseline FP-CIT. The other 2 patients with positive FP-CIT and negative MIBG had no history of cerebrovascular disease. One patient with normal baseline/follow up FP-CIT scans and positive MIBG (HMR 1.52) had type 2 diabetes and was on amlodipine, both of which may have contributed to the abnormal MIBG uptake, although the uptake does appear absent visually, rather than reduced. The other 2 patients with normal baseline FP-CIT scans and positive MIBG (HMR values of 1.44 and 0.98 ) were on no interacting medications and had no relevant medical history; both had positive follow-up FP-CIT.

The proportion of patients with MCI-LB with positive MIBG and proportion with positive FP-CIT, broken down into those with each of the 4 core LB features, is given in table 4 . Fisher exact test showed no significant difference in proportion of positive MIBG scans vs proportion of positive FPCIT scans for any core feature.

\section{Discussion}

In this prospective study of cardiac sympathetic imaging in MCI, the sensitivity for detecting MCI-LB was 59\%, with $95 \%$ $\mathrm{CI}$ of $42 \%-75 \%$; that is, around half to 3 quarters of patients over 60 with probable MCI-LB have sympathetic denervation detectable using cardiac I-123-MIBG scintigraphy. The specificity was $88 \%$ (95\% CI, 75\%-96\%), which is above the conventional $80 \%$ cutoff for diagnostic utility in specialist settings, ${ }^{17}$ further supported by the positive likelihood ratio of 5.1. The estimated diagnostic accuracy of $75 \%(64 \%-84 \%)$ is similar to the results in our earlier smaller study using FP-CIT. ${ }^{5}$ The strengths of our study include the prospective design and thorough consensus clinical assessment, and the use of FP-CIT imaging, resulting in high confidence in the patient groups.

Use of cardiac MIBG would help identify people with LBD in MCI cohorts, thereby improving disease-specific stratification 
and enabling disease-modifying therapies to focus on the relevant target disease. Early identification could also allow for earlier symptomatic intervention and planning, keeping those patients with MCI at high risk of converting to DLB under regular medical review, and could enable patients with early disease to enter disease-modifying clinical trials. Studies conducted at the dementia stage show greater sensitivity than our study, with similar or slightly higher specificity. The multicenter study of Yoshita et al. ${ }^{18}$ reported sensitivity of $69 \%$ and specificity of $89 \%$. There have been no multicenter studies carried out in the UK, where the population would be similar to ours. Our previous single-center pilot study investigating cardiac MIBG at the dementia stage reported a $71 \%$ sensitivity and specificity of $81 \% .{ }^{19}$ The specificity at the earlier MCI stage therefore appears similar to the dementia stage, although cohorts are modest in both studies. Although the specificity of $88 \%$ is high, the low prior probability of a patient having MCI-LB outside a specialist setting means that in practice both cardiac MIBG and FP-CIT are only suitable tests for patients where there is good reason to suspect they may have LBD. It would, for example, not be appropriate to screen a general group of patients with MCI for MCI-LB with either biomarker as many false-positives would arise, even with the high specificity.

Two of our older adults with normal cognition had visually abnormal MIBG scans, with HMR results well below the mean of the remaining 30 controls. Two other controls had FP-CIT scans rated as abnormal. It is recognized that occult LBD (usually termed incidental LBD) is present in many older people, ${ }^{20}$ which may explain these findings. A study of human myocardial tissue found incidental LB pathology in 1 / 23 healthy controls aged $70 \pm 4$ years. $^{21}$

As an exploratory analysis, we recategorized the MCI groups by core features only to provide a fair comparison between MIBG and FP-CIT. The overall accuracies were similar for the biomarkers at $79 \%$ for MIBG (95\% CI, 68\%-87\%) and $76 \%$ for FP-CIT (95\% CI, 65\%-85\%). In this study, 3 patients with probable MCI-LB, defined as those with 2 or more core LB symptoms, had abnormal cardiac MIBG with normal FP-CIT and 3 vice versa. There are arguments for using cardiac MIBG over FP-CIT in some clinical scenarios (e.g., where frontotemporal dementia is a differential diagnosis), and FP-CIT over MIBG in others (e.g., in a person with heart failure), which needs further careful investigation with a larger study. Which biomarker, if any, should be the first-line investigation in clinical cases of suspected MCI-LB is an important unanswered question in this field. Our data suggest that although there is no significant difference in overall diagnostic accuracy, the 2 scans give a different result in around 1 in 4 cases with LB symptoms. Combining the 2 scans routinely is not recommended as this affects the specificity. The symptom patterns do not suggest which type of clinical presentation might favor choosing one biomarker over the other in clinical practice, although numbers are modest here.
Limitations of our study include the use of consensus diagnosis as gold standard, rather than histopathology following death. However, thus far 5 MCI participants have died and had autopsy assessments. Two with probable MCI-LB both had neocortical LBD, and 3 with MCI-AD all met standard criteria for $\mathrm{AD}$ (including all Braak stages 5 and 6). This provides some early validation for our diagnoses. Also, the specificity may be higher as our patients with MCI-AD may have LBD that is not yet manifest in any core symptoms or on FP-CIT imaging. Other studies have demonstrated that a significant proportion of participants with a clinical diagnosis of $\mathrm{AD}$ have some $\mathrm{LB}$ pathology postmortem; for example, $46 \%$ of $\mathrm{AD}$ cases in the initial Alzheimer's Disease Neuroimaging Initiative autopsy cohort had a coincident pathologic diagnosis of DLB. ${ }^{2}$ We recognize that well-characterized clinical cohorts of MCI-AD will contain a small proportion of participants with non- $\mathrm{AD}$ pathology; this does not undermine the conclusion that MIBG is a specific marker for LBD. Although our final sample of 43 patients with MCI-AD and 37 patients with probable MCI-LB was slightly lower than our original sample size estimate, we were still able to estimate diagnostic accuracy to within \pm 10 percentage points.

We included patients with cardiovascular risk factors, those with diabetes, and those with known mild heart disease, so that our population would be a representative sample of older people. Myocardial infarction within 1 year of recruitment or heart failure of class II or worse were exclusion criteria. Volunteers were not excluded if they had risk factors for cardiac disease, or less severe heart failure, because we aimed to assess MIBG in a real-world setting, where diabetes and risk factors for cardiac disease are extremely common. However, a detailed medical history including assessment of cardiac risk factors was taken so we could examine the effect of these. We also included those on some potentially interacting medications, stopping only those recommended by Jacobson and Travin. ${ }^{8}$ Our results, and that of our pilot study in DLB, ${ }^{19}$ show no good evidence to suggest this had a major effect on HMR or on the diagnostic accuracy but the numbers are too small for formal analysis to demonstrate this.

The results of this single-center study support the 2020 consensus recommendations on the diagnosis of MCI-LB, providing evidence that cardiac MIBG is useful in clinical practice even at the MCI stage, with an abnormal scan highly suggestive of MCI-LB. These results show that further work in a multicenter setting is justified.

\section{Acknowledgment}

The authors thank the people living with MCI, the healthy older adults, and the family members who participated in this study; Helen Kain for ongoing support with patient liaison and data entry; the Nuclear Medicine department at the Newcastle upon Tyne Hospitals NHS Foundation Trust for scanning the participants; and the staff of the NIHR Clinical Research Network North East and Cumbria for their support with participant recruitment. Authors at Newcastle University 
are supported by the NIHR Newcastle Biomedical Research Centre awarded to the Newcastle Hospitals NHS Foundation Trust and Newcastle University. John O'Brien is supported by the NIHR Cambridge Biomedical Research Centre. The authors acknowledge support for this investigator-led study from GE Healthcare, which provided ligand for the FP-CIT scans. GE Healthcare had no involvement in this study.

\section{Study Funding}

This study was funded by a major project research grant from Alzheimer's Research UK (ARUK-PG2015-13).

\section{Disclosure}

G.R. has received honoraria from GE Healthcare for delivering educational workshops on FP-CIT imaging. G.S.P. has received honoraria from GE Healthcare for delivering educational workshops on FP-CIT imaging and fees for backup reporting from GE Healthcare. J.-P.T. has received honoraria from GE Healthcare for delivering educational presentations on Lewy body disease and has consulted for Sosei-Heptares and Kyowa-Kirin. J.O. has acted as a consultant for Axon Neuroscience, TauRx, GE Healthcare, Lilly, and Eisai; has been a recipient of grant support from Alliance Medical, GE Healthcare, and Merck; and received honoraria for talks for GE Healthcare. A.J.T. has received support for investigator led studies and honoraria from GE Healthcare. R.D., P.C.D., S.L., J.C., C.A.H., S.J.C., M.J.F., L.A., N.B., S.B., K.H., T.A., and J.L. declare that they have no relevant conflicts of interest to disclose. Go to Neurology.org/ $\mathrm{N}$ for full disclosures.

\section{Publication History}

Received by Neurology August 3, 2020. Accepted in final form March 11, 2021.

\section{Appendix Authors}

\begin{tabular}{|c|c|c|}
\hline Name & Location & Contribution \\
\hline $\begin{array}{l}\text { Gemma } \\
\text { Roberts, } \\
\text { PhD }\end{array}$ & $\begin{array}{l}\text { Newcastle University/The } \\
\text { Newcastle upon Tyne } \\
\text { Hospitals NHS Foundation } \\
\text { Trust }\end{array}$ & $\begin{array}{l}\text { Major role in the acquisition of } \\
\text { data, analysis and } \\
\text { interpretation of the data, } \\
\text { drafted the manuscript }\end{array}$ \\
\hline $\begin{array}{l}\text { Rory } \\
\text { Durcan, } \\
\text { MRCPI, } \\
\text { MSc }\end{array}$ & Newcastle University & $\begin{array}{l}\text { Major role in the acquisition of } \\
\text { data, analysis and } \\
\text { interpretation of the data }\end{array}$ \\
\hline $\begin{array}{l}\text { Paul C. } \\
\text { Donaghy, } \\
\text { PhD }\end{array}$ & Newcastle University & $\begin{array}{l}\text { Major role in the acquisition of } \\
\text { data, analysis and } \\
\text { interpretation of the data, } \\
\text { revising the manuscript for } \\
\text { intellectual content }\end{array}$ \\
\hline $\begin{array}{l}\text { Sarah } \\
\text { Lawley, } \\
\text { MBBS }\end{array}$ & Newcastle University & $\begin{array}{l}\text { Major role in the acquisition of } \\
\text { data }\end{array}$ \\
\hline $\begin{array}{l}\text { Joanna } \\
\text { Ciafone, } \\
\text { PhD }\end{array}$ & Newcastle University & $\begin{array}{l}\text { Major role in the acquisition of } \\
\text { data }\end{array}$ \\
\hline
\end{tabular}

\section{Appendix (continued)}

\begin{tabular}{|c|c|c|}
\hline Name & Location & Contribution \\
\hline $\begin{array}{l}\text { Calum A. } \\
\text { Hamilton, } \\
\text { MSc }\end{array}$ & Newcastle University & $\begin{array}{l}\text { Major role in the acquisition of } \\
\text { data }\end{array}$ \\
\hline $\begin{array}{l}\text { Sean J. } \\
\text { Colloby, } \\
\text { PhD }\end{array}$ & Newcastle University & $\begin{array}{l}\text { Major role in the acquisition of } \\
\text { data, analysis and } \\
\text { interpretation of the data }\end{array}$ \\
\hline $\begin{array}{l}\text { Michael } \\
\text { Firbank, } \\
\text { PhD }\end{array}$ & Newcastle University & $\begin{array}{l}\text { Designed and conceptualized } \\
\text { study, analysis and } \\
\text { interpretation of the data, } \\
\text { revising the manuscript for } \\
\text { intellectual content }\end{array}$ \\
\hline $\begin{array}{l}\text { Louise } \\
\text { Allan, PhD }\end{array}$ & University of Exeter & $\begin{array}{l}\text { Designed and conceptualized } \\
\text { study, revising the manuscript } \\
\text { for intellectual content }\end{array}$ \\
\hline $\begin{array}{l}\text { Nicola } \\
\text { Barnett, } \\
\text { MSc }\end{array}$ & Newcastle University & $\begin{array}{l}\text { Major role in the acquisition of } \\
\text { data }\end{array}$ \\
\hline $\begin{array}{l}\text { Sally } \\
\text { Barker, } \\
\text { BSc }\end{array}$ & Newcastle University & $\begin{array}{l}\text { Major role in the acquisition of } \\
\text { data }\end{array}$ \\
\hline $\begin{array}{l}\text { Kim } \\
\text { Howe, BSc }\end{array}$ & $\begin{array}{l}\text { The Newcastle upon Tyne } \\
\text { Hospitals NHS Foundation } \\
\text { Trust }\end{array}$ & $\begin{array}{l}\text { Major role in the acquisition of } \\
\text { data }\end{array}$ \\
\hline $\begin{array}{l}\text { Tamir Ali, } \\
\text { FRCR }\end{array}$ & $\begin{array}{l}\text { The Newcastle upon Tyne } \\
\text { Hospitals NHS Foundation } \\
\text { Trust }\end{array}$ & $\begin{array}{l}\text { Major role in the acquisition of } \\
\text { data }\end{array}$ \\
\hline
\end{tabular}

George S. The Newcastle upon Tyne

Petrides, Hospitals NHS Foundation

Designed and conceptualized study, major role in the acquisition of data, analysis and interpretation of the data, revising the manuscript for intellectual content

Jim Lloyd, Newcastle University/The PhD Newcastle upon Tyne Hospitals NHS Foundation Trust

Designed and conceptualized study, major role in the acquisition of data, analysis and interpretation of the data, revising the manuscript for intellectual content

\begin{tabular}{|c|c|c|}
\hline $\begin{array}{l}\text { John-Paul } \\
\text { Taylor, } \\
\text { PhD }\end{array}$ & Newcastle University & $\begin{array}{l}\text { Designed and conceptualized } \\
\text { study, major role in the } \\
\text { acquisition of data, analysis } \\
\text { and interpretation of the data, } \\
\text { revising the manuscript for } \\
\text { intellectual content }\end{array}$ \\
\hline $\begin{array}{l}\text { John } \\
\text { O'Brien, } \\
\text { DM }\end{array}$ & University of Cambridge & $\begin{array}{l}\text { Designed and conceptualized } \\
\text { study, analysis and } \\
\text { interpretation of the data, } \\
\text { revising the manuscript for } \\
\text { intellectual content }\end{array}$ \\
\hline $\begin{array}{l}\text { Alan J. } \\
\text { Thomas, } \\
\text { PhD }\end{array}$ & Newcastle University & $\begin{array}{l}\text { Designed and conceptualized } \\
\text { study, major role in the } \\
\text { acquisition of data, analysis } \\
\text { and interpretation of the data, } \\
\text { revising the manuscript for } \\
\text { intellectual content }\end{array}$ \\
\hline
\end{tabular}

\section{References}

1. Jack CR Jr, Bennett DA, Blennow K, et al. NIA-AA Research Framework: toward a biological definition of Alzheimer's disease. Alzheimers Dement. 2018;14(4):535-562.

2. Toledo JB, Cairns NJ, Da X, et al. Clinical and multimodal biomarker correlates of ADNI neuropathological findings. Acta Neuropathol Commun. 2013;1:65. 
3. McKeith IG, Boeve BF, Dickson DW, et al. Diagnosis and management of dementia with Lewy bodies: fourth consensus report of the DLB Consortium. Neurology. 2017; 89(1):88-100.

4. McKeith I, Ferman T, Thomas AJ, et al. Research criteria for the diagnosis of prodromal dementia with Lewy bodies. Neurology. 2020;94(17):743-755.

5. Thomas AJ, Donaghy P, Roberts G, et al. Diagnostic accuracy of dopaminergic imaging in prodromal dementia with Lewy bodies. Psychol Med. 2019;49(3):396-402.

6. Cohen JF, Korevaar DA, Altman DG, et al. STARD 2015 guidelines for reporting diagnostic accuracy studies: explanation and elaboration. BMJ Open. 2016;6(11):e012799.

7. Donaghy PC, Ciafone J, Durcan R, et al. Mild cognitive impairment with Lewy bodies: neuropsychiatric supportive symptoms and cognitive profile. Psychol Med. 2020:1-9.

8. Jacobson AF, Travin MI. Impact of medications on MIBG uptake, with specific attention to the heart: comprehensive review of the literature. J Nucl Cardiol. 2015;22(5):980-993.

9. Albert MS, DeKosky ST, Dickson D, et al. The diagnosis of mild cognitive impairment due to Alzheimer's disease: recommendations from the National Institute on AgingAlzheimer's Association workgroups on diagnostic guidelines for Alzheimer's disease. Alzheimers Dement. 2011;7(3):270-279.

10. McKeith I, O’Brien J, Walker Z, et al. Sensitivity and specificity of dopamine transporter imaging with (123)I-FP-CIT SPECT in dementia with Lewy bodies: a phase III, multicentre study. Lancet Neurol. 2007;6:305-313.

11. McKeith I, Ballard C, O’Brien J, et al. Predictive accuracy of clinical diagnostic criteria for dementia with Lewy bodies: a prospective neuropathological validation study. Neurology. 2000;54(7):1050-1058.

12. Roberts G, Kane JPM, Lloyd JJ, et al. A comparison of visual and semiquantitative analysis methods for planar cardiac 123I-MIBG scintigraphy in dementia with Lewy bodies. Nucl Med Commun. 2019;40(7):734-743.
13. Roberts G, Lloyd JJ, Kane JPM, et al. Cardiac (123)I-MIBG normal uptake values are population-specific: results from a cohort of controls over 60 years of age. $\mathrm{J} \mathrm{Nucl}$ Cardiol Epub 2019 Sep 16.

14. Benamer TS, Patterson J, Grosset DG, et al. Accurate differentiation of parkinsonism and essential tremor using visual assessment of [123I]-FP-CIT SPECT imaging: the [123I]-FP-CIT study group. Mov Disord. 2000;15(3):503-510.

15. O’Brien JT, Colloby S, Fenwick J, et al. Dopamine transporter loss visualized with FPCIT SPECT in the differential diagnosis of dementia with Lewy bodies. Arch Neurol. 2004;61(6):919-925

16. Morbelli S, Esposito G, Arbizu J, et al. EANM practice guideline/SNMMI procedure standard for dopaminergic imaging in Parkinsonian syndromes 1.0. Eur J Nucl Med Mol Imaging. 2020;47(8):1885-1912.

17. Postuma RB, Berg D, Stern M, et al. MDS clinical diagnostic criteria for Parkinson's disease. Mov Disord. 2015;30(12):1591-1601.

18. Yoshita M, Arai $\mathrm{H}$, Arai $\mathrm{H}$, et al. Diagnostic accuracy of 123I-metaiodobenzylguanidine myocardial scintigraphy in dementia with Lewy bodies: a multicenter study. PLoS One. 2015;10(3):e0120540.

19. Kane JPM, Roberts G, Petrides GS, Lloyd JJ, O’Brien JT, Thomas AJ. ${ }^{123} \mathrm{I}-\mathrm{MIBG}$ scintigraphy utility and cut-off value in a clinically representative dementia cohort. Parkinsonism Relat Disord. 2019;62:79-84.

20. Beach TG, Adler CH, Sue LI, et al. Multi-organ distribution of phosphorylated alphasynuclein histopathology in subjects with Lewy body disorders. Acta Neuropathol. 2010;119(6):689-702.

21. Goldstein DS, Sullivan P, Holmes C, Miller GW, Sharabi Y, Kopin IJ. A vesicular sequestration to oxidative deamination shift in myocardial sympathetic nerves in Parkinson's disease. J Neurochem. 2014;131(2):219-228. 


\section{Neurology}

\section{Accuracy of Cardiac Innervation Scintigraphy for Mild Cognitive Impairment With Lewy Bodies}

Gemma Roberts, Rory Durcan, Paul C. Donaghy, et al.

Neurology 2021;96;e2801-e2811 Published Online before print April 21, 2021

DOI 10.1212/WNL.0000000000012060

This information is current as of April 21, 2021

\section{Updated Information \&} Services

References

Subspecialty Collections

Permissions \& Licensing

Reprints including high resolution figures, can be found at: http://n.neurology.org/content/96/23/e2801.full

This article cites 19 articles, 4 of which you can access for free at: http://n.neurology.org/content/96/23/e2801.full\#ref-list-1

This article, along with others on similar topics, appears in the following collection(s):

Class I

http://n.neurology.org/cgi/collection/class_1

Dementia with Lewy bodies

http://n.neurology.org/cgi/collection/dementia_with_lewy_bodies

MCI (mild cognitive impairment)

http://n.neurology.org/cgi/collection/mci_mild_cognitive_impairment

Parkinson's disease/Parkinsonism

http://n.neurology.org/cgi/collection/parkinsons_disease_parkinsonism

Information about reproducing this article in parts (figures,tables) or in its entirety can be found online at:

http://www.neurology.org/about/about_the_journal\#permissions

Information about ordering reprints can be found online:

http://n.neurology.org/subscribers/advertise

Neurology ${ }^{\circledR}$ is the official journal of the American Academy of Neurology. Published continuously since 1951, it is now a weekly with 48 issues per year. Copyright Copyright ( 2021 The Author(s). Published by Wolters Kluwer Health, Inc. on behalf of the American Academy of Neurology.. All rights reserved. Print ISSN: 0028-3878. Online ISSN: 1526-632X.



